admissions for general primary gastrointestinal diagnoses (excluding DD) during the same period. The proportion of DD admissions by age remained largely consistent, but over the 14 years, more women were admitted than men ( $61 \%$ vs $39 \%$ ), most notably in those over 50 years $\left(65,571\right.$ 우 vs $\left.39,558 \delta^{\lambda}\right)$. Mean LOS for all cases decreased from 6.4 to 3.2 days and mean inpatient LOS from 10.6 to 8.8 days.

The rise in DD admissions was almost entirely due to admissions $\leq 1$ day (2562 in 1996-1997, 7893 in 2009-2010). Of these, $74 \%$ related to diagnostic colonoscopy/sigmoidoscopy (2158 in 1996-1997, 6089 in 2009-2010). Patients were most often admitted under General Surgery (71\%), Gastroenterology $(16 \%)$ or General Medicine (10\%).

Of 7893 admissions $\leq 1$ day in 2009-2010, 6089 were for colonoscopy/sigmoidoscopy, and 91\% (7186) had an admission reason recorded. Acute medical care or treatment was coded in 69\% (3822) of colonoscopy episodes and 67\% (1100) of other episodes, with investigation only coded in 31\% (1711) and $33 \%$ (553), respectively. Only $2.7 \%$ of admissions $\leq 1$ day were emergency, with $15 \%$ undergoing colonoscopy.

Conclusion Admissions for DD in Scotland increased considerably between 1996 and 2010. Most of this rise is attributable to admissions $\leq 1$ day for colonoscopy/sigmoidoscopy, but $26 \%$ of the rise in admissions $\leq 1$ day was attributable to nondiagnostic admissions where acute treatment was undertaken, adding to the healthcare burden.

Competing interests I. Arnott Grant / Research Support from: Shire, H. Paterson Grant / Research Support from: Shire, R. J. Nicholls Grant / Research Support from: Shire, A. Crowe Grant / Research Support from: Shire, A. Knight Grant / Research Support from: Shire, D. Clark Grant / Research Support from: Shire, J. Bauer Grant / Research Support from: Shire, L. Yen Employee of: Shire, P. Hodgkins Employee of: Shire, M. Dunlop Grant / Research Support from: Shire.

Keywords burden, diverticulitis, epidemiology.

\section{OC-013 A DOUBLING OF ADMISSIONS DUE TO DIVERTICULAR DISEASE IN SCOTTISH HOSPITALS, IN THE LAST 14 YEARS}

\author{
doi:10.1136/gut.2011.239301.13
}

I Arnott, ${ }^{1 *}$ H Paterson, ${ }^{1}$ R J Nicholls, ${ }^{2}$ A Crowe, ${ }^{3}$ A Knight, ${ }^{3}$ D Clark, ${ }^{4}$ J Bauer, ${ }^{4}$ L Yen, ${ }^{5}$ P Hodgkins, ${ }^{5}$ M Dunlop' 1 University of Edinburgh, Western General Hospital, Edinburgh, UK; ${ }^{2}$ Imperial College, St Mary's Campus, London, UK; ${ }^{3}$ Corvus, Buxted, UK; ${ }^{4}$ Information Services Division, NHS National Services Scotland, Edinburgh, UK; ${ }^{5}$ Global Health Economics \& Outcomes Research, Shire Pharmaceuticals, Wayne, USA

Introduction Information on the epidemiology and burden of diverticular disease (DD) requiring hospitalisation is limited. The study reviewed DD admissions in Scotland in the last 14 years.

Methods The Scottish national record linkage database was used to assess day case and inpatient hospital admissions from April1 1996 to 31 March 2010 for DD (ICD10 K57). Cases selected were Scottish residents with coding of DD as primary diagnosis on the Scottish Morbidity Record (SMR01). Continuous inpatient stays (CIS) were assessed to include intra-department and inter-hospital transfers. Age, gender and length of stay (LOS) associated with DD were compared to admissions for other gastrointestinal problems in the same period.

Results Hospital admissions with a primary DD diagnosis increased by $107 \%$ over the 14 years (5284 in 1996-1997 to 10,935 in $2009-2010$ ), compared with a $20 \%$ increase in 